Relations industrielles

Industrial Relations

\title{
Diane-Gabrielle Tremblay, L'emploi en devenir
}

\section{Colette Bernier}

Volume 47, numéro 1, 1992

URI : https://id.erudit.org/iderudit/050757ar

DOI : https://doi.org/10.7202/050757ar

Aller au sommaire du numéro

Éditeur(s)

Département des relations industrielles de l'Université Laval

ISSN

0034-379X (imprimé)

1703-8138 (numérique)

Découvrir la revue

Citer ce compte rendu

Bernier, C. (1992). Compte rendu de [Diane-Gabrielle Tremblay, L'emploi en devenir]. Relations industrielles / Industrial Relations, 47(1), 174-176.

https://doi.org/10.7202/050757ar

Tous droits réservés (C Département des relations industrielles de l'Université Laval, 1992
Ce document est protégé par la loi sur le droit d'auteur. L'utilisation des services d'Érudit (y compris la reproduction) est assujettie à sa politique d'utilisation que vous pouvez consulter en ligne.

https://apropos.erudit.org/fr/usagers/politique-dutilisation/ 
d'accroître la capacité des organisations de s'adapter à un environnement de moins en moins stable et prévisible.

La présentation des cas obéit à la séquence habituelle qui caractérise toute intervention auprès des organisations qui utilise l'approche DO: 1) les contacts initiaux et la nature du contrat; 2) le diagnostic organisationnel; 3) l'intervention proprement dite ou la séquence des actions porteuses d'un changement; 4) enfin, le suivi ou l'enracinement des changements effectués. La majorité des cas répertoriés viennent illustrer les étapes diagnostic et intervention qui constituent le coeur de tout programme de changement organisationnel. Des actions, de type «formation d'équipe de travail» (teambuilding), de rencontre de confrontation (confrontation me日ting), d'enquête avec rétroaction d'information aux intéressés (survey feedback), de diagnostic d'environnement externe en termes de menaces et d'opportunités sont abondamment illustrées.

Chaque cas est suivi d'une série de questions auxquelles l'étudiant en DO ou le futur intervenant cherche à apporter des réponses. C'est ainsi qu'en plus de se familiariser avec les concepts et les principes du DO, le futur consultant aura l'occasion d'acquérir les habiletés de diagnostic et de programmation d'actions pertinentes. Il faut également signaler que ces cas sont tirés à la fois de situations vécues aussi bien dans les organisations du secteur public (hôpital, centre communautaire, diocèse) que du secteur privé. De plus, les auteurs, pour la rédaction des cas, ont fait appel aux cadres supérieurs des organisations et aux consultants qui ont vécu les situations décrites. On se retrouve donc avec un premier ouvrage du genre dans le domaine du DO, alors qu'on sait que de tels recueils de cas existent depuis très longtemps dans les autres domaines de la gestion des organisations. Il s'agit donc d'un instrument précieux pour ceux et celles qui dispensent un enseignement dans ce domaine ou encore qui le pratiquent.

Laurent BÉLANGER

Université Laval

L'emploi en devenir, par Diane-Gabrielle Tremblay, Québec, Institut québécois de recherche sur la culture, collection Diagnostic, 1990, 121 p., ISBN 2-89224-141-3

La collection «Diagnostic" dans faquelle est paru le livre de Diane-Gabrielle Tremblay réunit des ouvrages portant sur des questions d'actualité et qui sont destinés au grand public. Diagnostic veut informer, provoquer la réflexion, stimuler la recherche et aider le lecteur à se former une opinion éclairée. Ce petit livre d'une centaine de pages portant sur ce que certains ont appelé les nouvelles formes d'emploi, d'autres les formes atypiques ou précaires d'emplois, remplira certainement ce rôle. Il présente, dans un langage simple, les principales formes d'emplois atypiques actuellement en progression au Québec (emplois à la pige, à temps partiel, occasionnels, à contrat, etc.) et les nouvelles théories économiques (théories de la régulation, thèses de la segmentation du marché du travail, etc.) qui tentent d'expliquer le phénomène. 
Le premier chapitre montre en quoi ces nouvelles formes d'emplois renvoient à un marché du travail passablement différent de ce qu'il était dans les années cinquante à soixante-dix. Dans ces années-là, la norme était aux emplois permanents, à temps plein, aux emplois comportant une certaine sécurité d'emploi et divers avantages sociaux. Or, depuis le milieu des années soixante-dix, de nouvelles formes d'emplois présentant souvent une certaine précarité tendent à devenir plus nombreuses et à constituer, en quelque sorte, la nouvelle norme d'emploi. L'auteure réussit à présenter, de façon relativement simple, les différents critères de définition de ces nouvelles formes d'emplois (durée de l'emploi, régularité du travail, caractère unique de l'employeur) tels qu'établis par diverses études mais sans buter les lecteurs et lectrices aux nombreuses références qu'on retrouve dans les ouvrages académiques. Pourtant, quelques références auraient été souhaitables, pour les étudiants ou autres personnes voulant poursuivre ou approfondir la recherche.

Au deuxième chapitre, l'auteure tente de replacer le phénomène de la précarisation des emplois dans les grands débats théoriques de l'heure. D'abord, celui entourant la recherche de flexibilité de la part des entreprises. Ensuite, celui opposant deux visions du marché du travail: la vision keynésienne, dominante jusqu'à tout récemment, et la vision néo-classique qui réapparait avec la crise de l'Etat-providence. Après avoir défini les principales formes de flexibilité, l'auteure jette un coup d'oeil rapide sur les modèles développés dans différents pays ('Allemagne, la Suède, l'Australie). On reste pourtant sur notre faim quand l'auteure aborde le Québec en disant que les formules d'ajustement aux nouvelles réalités y sont beaucoup moins développées que dans ces pays. De même, quand abordant l'analyse des marchés internes de travail et, par ce biais, les questions de formation et de culture d'entreprise, l'auteure tente quelques généralisations sur la situation québécoise et canadienne. Mais bien que la description de la réalité québécoise laisse à désirer, il faut dire que la tâche que s'est donnée l'auteure de présenter de nouvelles théories économiques à l'intention des non-initiés n'était pas chose facile et elle y a réussi.

Les deux chapitres suivants offrent par ailleurs un portrait bien documenté de la situation de l'emploi ainsi que des formes particulières d'emploi au Québec et au Canada. Le chapitre trois y décrit le contexte du chômage où la situation, sans être aussi mauvaise que celle de certains pays européens, est nettement moins bonne que celle des États-Unis. Au chapitre quatre, sur les nouvelles formes d'emploi, l'auteure apporte des informations récentes sur le travail à temps partiel, la seule forme d'emploi particulière sur laquelle nous ayons des données statistiques précises au Canada. Sur le travail temporaire, à contrat ou encore le travail autonome, tout porte à croire, selon l'auteure, que ces formes d'emplois aient aussi beaucoup progressé au cours des dernières années. Malgré le caractère visiblement sérieux de la recherche statistique effectuée par l'auteure, on peut par contre regretter que le style adopté l'ait amenée à ne pas citer systématiquement ses sources. On peut aussi regretter que ces deux chapitres qui voulaient présenter la réalité des nouvelles formes d'emplois n'aient en aucune façon fait appel aux études québécoises présentant les conditions de vie et de travail de ces salariés, hommes et femmes. Ceci aurait sans doute contribué à rendre le texte plus facile à lire et à sortir l'analyse d'une vision, à notre avis, trop strictement économiste et statistique. 
Enfin, le dernier chapitre tente d'amorcer une réflexion afin d'identifier des pistes de solution alternatives à la stratégie de précarisation des emplois. Encore une fois, l'auteure présente de façon simple et claire les théories économiques (malheureusement, encore une fois, sans références systématiques) qui peuvent servir à identifier les stratégies possibles. Ici sont amenées les thèses sur la segmentation des marchés du travail qui montrent les risques d'un éclatement du marché du travail entre les «bons» et les «mauvais» emplois. En présentant les modèles traditionnels de gestion de main-d'oeuvre encore dominants dans les grandes entreprises (le système d'emploi industriel et le système d'emploi salarié), l'auteure met le doigt sur différentes pratiques (telles la règle de l'ancienneté ou encore la règle de la sécurité d'emploi) qui viennent en porte-à-faux à la recherche de flexibilité des entreprises. Face à cela, l'auteure suggère, en conclusion, une stratégie axée sur la formation professionnelle et la mobilité des personnes en emploi. Ce dernier chapitre aurait pu mieux cibler, à notre avis, les pistes de solutions possibles s'il avait clairement identifié les propositions amenées par les différents groupes sociaux actifs sur la question de l'emploi dans la société québécoise actuelle.

Notre principale critique de ce livre est d'ailleurs d'avoir présenté les entreprises comme le seul moteur des stratégies d'emploi; les syndicats et les autres groupes sociaux, comme le aForum pour l'emploi», sont absolument absents du modèle d'analyse. Ceci n'est pas sans laisser un malaise quand on sait que se dessine au Québec un modèle original de concertation sur les questions reliées à l'emploi que commencent à envier les autres provinces canadiennes. Aussi doit-on considérer ce livre pour ce qu'il est: un livre qui fait état de façon simple et condensée des principales théories économiques liées à la question de l'emploi; un livre qui expose bien les statistiques récentes sur les nouvelles formes d'emploi; un livre qui, par sa présentation bien articulée des théories de l'emploi, peut provoquer une réflexion stimulante; un livre cependant où les stratégies des acteurs sociaux sont absentes et où donc l'analyse concrète de l'évolution du marché de l'emploi au Québec laisse à désirer. Malgré cette lacune, c'est un livre à lire pour qui veut comprendre les grandes tendances de l'emploi.

Colette Bernier

Université Laval

School Teaching in Canada, by Alexander Lockhart, Toronto, University of Toronto Press, 1991, 175 p., ISBN 0-8020-2748-2 (cloth) and ISBN 08020-67883 (paper)

Alexander Lockhart's informative and comprehensive survey of Canadian elementary and secondary school teachers is one of several studies of the profession in Canada initiated by the Analytical Studies Branch of Statistics Canada under the direction of Paul Reed. Among the topics discussed in this work are the characteristics of today's teachers, the conditions under which they work, their professional associations, career patterns in teaching, the political environment, current pedagogy, and the public interest. The resulting work goes far beyond the usual narrowly conceived analysis or profile of a single 